\title{
Predictive Value of Myocardial injury in Patients with COVID-19 Admitted to a Quaternary Hospital in the City of Rio de Janeiro
}

Antônio Sérgio Cordeiro da Rocha, ${ }^{(0)}$ Andre Volschan, ${ }^{(\infty)}$ Luiz Antonio Almeida Campos, Roberta Pereira dos Santos Coelho, Dominique Cardoso de Almeida Thielmann, Carlos Augusto Lobbe Cotta Ferreira, Alexandre Siciliano Colafranceschi

Hospital Pró-cardíaco, Rio de Janeiro, RJ - Brazil

\section{Abstract}

Background: In Brazil the factors involved in the risk of death in patients with COVID-19 have not been well established.

Objective: To analyze whether elevations of high-sensitivity troponin I (hTnI) levels influence the mortality of patients with COVID-19.

Methods: Clinical and laboratory characteristics of hospitalized patients with COVID-19 were collected upon hospital admission. Univariate and binary logistic regression analyzes were performed to assess the factors that influence mortality. P-value $<0.05$ was considered significant.

Results: This study analyzed192 patients who received hospital admission between March 16 and June 2, 2020 and who were discharged or died by July 2,2020 . The mean age was $70 \pm 15$ years, 80 (41.7\%) of whom were women. In comparison to those who were discharged, the 54 (28.1\%) who died were older ( $79 \pm 12$ vs $66 \pm 15$ years; $\mathrm{P}=0.004)$, and with a higher Charlson's index ( $5 \pm 2$ vs $3 \pm 2 ; \mathrm{P}=0.027$ ). More patients, aged $\geq 60$ years $(\mathrm{P}<0.0001)$, Charlson's inde $>1$ ( $\mathrm{P}=0.004)$, lung injury $>50 \%$ in chest computed tomography $(\mathrm{P}=0.011)$, with previous coronary artery disease $(\mathrm{P}=0.037)$, hypertension $(\mathrm{P}=0.033)$, stroke $(\mathrm{P}=0.008)$, heart failure $(\mathrm{P}=0.002)$, lymphocytopenia $(\mathrm{P}=0.024)$, high D-dimer $(\mathrm{P}=0.024)$, high INR $(\mathrm{P}=0.003)$, hTnI $(\mathrm{P}<0.0001)$, high creatinine $(\mathrm{P}<0.0001)$, invasive mechanical ventilation $(\mathrm{P}<0.0001)$, renal replacement therapy $(\mathrm{P}<0.0001)$, vasoactive amine $(\mathrm{P}<0.0001)$, and transfer to the ICU $(\mathrm{P}=0.001)$, died when compared to those who were discharged. In logistic regression analysis, elevated $\mathrm{hTnI}$ levels (OR=9.504; 95\% CI=1.281-70.528; $\mathrm{P}=0.028)$ upon admission, and the need for mechanical ventilation during hospitalization (OR=46.691; 95\% CI=2.360-923.706; $\mathrm{P}=0.012$ ) increased the chance of in-hospital mortality.

Conclusion: This study suggests that in COVID-19 disease, myocardial injury upon hospital admission is a harbinger of poor prognosis.

Keywords: COVID-19; SARS-CoV-2; Mortality; Myocardial Contusions; troponin I.

\section{Introduction}

Since the first reported cases in Wuhan, China, severe acute respiratory syndrome coronavirus 2 (SARS-CoV-2) has spread rapidly worldwide. According to the World Health Organization (WHO), on October 9, 2020, there were $36,754,395$ confirmed cases of COVID-19 in the world, with 1,064,838 deaths, while in Brazil there were $5,028,444$ confirmed cases with 148,957 deaths. ${ }^{1}$
Although SARS-CoV-2 explicitly refers to an aggression in the lungs, often inducing acute respiratory distress syndrome (ARDS), it also affects other organs, including blood vessels, brain, gastrointestinal tract, kidneys, heart, and liver, ${ }^{2}$ and cardiovascular complications are manifestations that contribute significantly to the mortality of these patients. ${ }^{3}$

Several biomarkers of myocardial injury are elevated in patients with COVID-19, and a rise in troponin levels 
have been associated with the prediction of malignant cardiac arrhythmias, ${ }^{4}$ and the need for mechanical ventilation $(\mathrm{MV})$. $^{3,5-8}$

This study sought to verify whether the elevation of high-sensitive troponin I (hTnI) levels upon hospital admission is an independent predictive factor of higher mortality in hospitalized patients with COVID-19.

\section{Methods}

This retrospective study involved a cohort of patients over 18 years of age who received hospital admission between March 16 and June 2, 2020, with a diagnosis of COVID-19, confirmed by nasopharyngeal reverse transcription-polymerase chain reaction (RT-PCR), or IgM and IgG measurement by chemiluminescent microplate immunoassay technique, and who were discharged or died by July 2, 2020.

The following characteristics were routinely collected upon hospital admission: sex, age, symptoms, comorbidities, blood count, biochemistry, prothrombin time with INR (INR), ultrasensitive PCR, D-dimer, simple X-ray, and chest computed tomography (CT) scan. hTnI (VITROS® High Sensitivity Troponin I assay), $\mathrm{N}$-terminal prohormone of brain natriuretic peptide (Roche NT-proBNP), fibrinogen, and transthoracic echocardiogram (TTE) were not collected in all patients upon hospital admission. The laboratory tests were those collected within 24 hours of the patients' admission.

Comorbidities and clinical manifestations were collected by the physician responsible for hospital admission and were described, non-systematically, in the research forms from which the data were extracted for analysis.

The need for invasive $\mathrm{MV}$, renal replacement therapy (RRT), extracorporeal membrane oxygenator (ECMO), the development of acute left ventricular systolic dysfunction (LVSD), or acute cor pulmonale, and the transfer to the intensive care unit (ICU) due to the worsening of the clinical condition during hospitalization, were also collected. Worsening of the clinical condition meant persistent desaturation, despite oxygenation by a high-flow nasal catheter, endotracheal intubation, resuscitation after cardiorespiratory arrest, and shock of any nature requiring vasoactive amines.

To analyze the chest CT scan, exams performed in the first 24 hours of hospitalization were included, and those with a "ground glass" infiltrate pattern, compatible with SARS-CoV-2 infection, ${ }^{9}$ were considered positive. All chest CT reports were reviewed by a senior radiologist, with an emphasis on compatible changes and the percentage of pulmonary parenchymal involvement.

TTE were performed following the recommendations of the Department of Cardiovascular Imaging (DIC, in Portuguese) of the Brazilian Society of Cardiology (SBC, in Portuguese) to perform cardiovascular imaging tests during the COVID-19 pandemic, ${ }^{10}$ prioritizing the analysis of the left ventricular systolic dysfunction (LVSD) and the presence of acute cor pulmonale. The degree of LVSD was assessed visually. These analyzes were performed within the first 48 hours of hospitalization, and all tests were reviewed and validated by a senior echocardiographer.

The review of the imaging exams was carried out without the doctors having any knowledge of the clinical, laboratory, or patient outcomes.

Symptoms and comorbidities were those reported by patients or provided by family members, caregivers, or companions depending on the degree of interaction of the patients at the time of hospital admission.

The comorbidities presented by the patients were assessed using the Charlson's comorbidity index. ${ }^{11}$

This study was approved by the institution's Ethics Committee (number 31543220.1.0000.5533).

\section{Statistical analysis}

Data were collected directly from the medical records by an independent clinical research team and subsequently included in the SPSS statistical package, version 22 (IBM SPSS $^{\circledR}$ ).

Continuous variables were exposed by mean and standard deviations, and categorical variables by numbers and the appropriate proportions. The unpaired Student $t$ test was used to compare continuous variables, and the Fisher's exact test or the Chi-square method was used to verify the existence of the association between categorical variables.

Binary logistic regression analysis was used to define which factors independently influenced mortality. To be included in this analysis, it was necessary that in the univariate analyses conducted by the Student's t test, Fisher exact test, or Chi-square test, the P-values were $\leq 0.05$. Two-tailed P-values $<0.05$ were considered statistically significant. 


\section{Results}

During the study period, 339 consecutive patients were admitted to cohort units for SARS-Cov-2 infection, of whom 205 had a confirmed diagnosis. Among the 205, 192 (93.6\%), who either died or were discharged by July 2, 2020, were included in this analysis.

Of the 192,80 (41.7\%) were women, aged $70 \pm 15$ years and Charlson's index of $3 \pm 2$. This study included 135 patients aged $\geq 60$ years $(70.3 \%)$, and $61(31.8 \%)$ were aged $\geq 80$ years. Charlson's index $>1$ was observed in 133 $(69.3 \%)$ patients.

During the study period, 54 (28.1\%) patients died.
Table 1 shows that, on average, deceased patients were 13 years older than those who were discharged and with a significantly higher Charlson's index.

Of the 54 who died, 27 (50\%) received palliative care during hospitalization. In 7 of the $27(25.9 \%)$, no invasive procedures, endotracheal intubation, renal replacement therapy, or resuscitation procedures were used, and the deaths occurred in semi-intensive care units.

As shown in Table 1, more patients of $\geq 60$ years, and with Charlson's index $>1$ died when compared to those who were discharged. Table 1 also shows that patients who died had more than $50 \%$ of the pulmonary parenchyma compromised, a previous history of

Table 1 - Factors involved in mortality upon hospital admission in a univariate analysis

\begin{tabular}{|c|c|c|c|c|c|}
\hline Characteristics & $\begin{array}{c}\text { Discharged } \\
(\mathrm{N}=138)\end{array}$ & Died $(\mathrm{N}=54)$ & p-value & OR & $95 \% \mathrm{CI}$ \\
\hline Age $\geq 60$ years, $N(\%)$ & $84(60.9)$ & $51(94.4)$ & $<0.0001$ & 10.929 & $3.247-36.779$ \\
\hline Charlson's index $>1, \mathrm{~N}(\%)$ & $121(87.7)$ & $54(100)$ & 0.004 & 1.446 & $1.310-1.547$ \\
\hline Men, N (\%) & $81(58.7)$ & $31(57.4)$ & 0.672 & 1.054 & $0.558-1.994$ \\
\hline Obesity, N (\%) & $37(26.8)$ & $14(25.9)$ & 1.0 & 0.995 & $0.467-1.954$ \\
\hline SAH, N (\%) & $79(57.2)$ & $40(74.1)$ & 0.033 & 2.134 & $1.064-4.279$ \\
\hline Diabetes, N (\%) & $48(34.8)$ & $20(37.0)$ & 0.867 & 1.103 & $0.573-2.121$ \\
\hline CAD, N (\%) & $26(18.8)$ & $18(33.3)$ & 0.037 & 2.154 & $1.060-4.375$ \\
\hline Stroke, N (\%) & $7(5.1)$ & $10(18.5)$ & 0.008 & 4.253 & $1.527-11.848$ \\
\hline COPD, N (\%) & $11(8.0)$ & $8(14.8)$ & 0.181 & 2.008 & $0.760-5.303$ \\
\hline CHF, N (\%) & $4(2.9)$ & $9(16.7)$ & 0.002 & 6.700 & $1.968-22.814$ \\
\hline CRF, N (\%) & $7(5.1)$ & $6(11.1)$ & 0.197 & 2.339 & $0.749-7.310$ \\
\hline $\mathrm{CT}$ pulmonary $>50 \%, \mathrm{~N}(\%)$ & $30(21.9)$ & $22(41.5)$ & 0.011 & 2.531 & $1.282-4.997$ \\
\hline Leukocytosis, N (\%) & $16(11.6)$ & $11(20.4)$ & 0.164 & 1.951 & $0.840-4.530$ \\
\hline Lymphocytopenia, N (\%) & $63(45.7)$ & $35(64.8)$ & 0.024 & 2.193 & 1.1434 .206 \\
\hline Thrombocytopenia, N (\%) & $44(31.9)$ & $21(38.9)$ & 0.398 & 1.360 & $0.707-2.614$ \\
\hline D-dimer $\geq 500 \mathrm{ng} / \mathrm{mL}, \mathrm{N}(\%)$ & $108(80.0)$ & $48(91.4)$ & 0.024 & 4.000 & $1.157-13.827$ \\
\hline hsRCP-t $>0.5 \mathrm{ng} / \mathrm{mL}, \mathrm{N}(\%)$ & $135(97.8)$ & $54(100)$ & 0.560 & 0.978 & $0.954-1.003$ \\
\hline hTnI $\geq 34 \mathrm{pg} / \mathrm{mL}, \mathrm{N}(\%)$ & $11(10.8)$ & $18(48.6)$ & $<0.0001$ & 7.837 & $3.192-19.345$ \\
\hline INR>1.3, N (\%) & $21(15.3)$ & $19(35.8)$ & 0.003 & 3.087 & $1.489-6.398$ \\
\hline Creatinine $>1.3 \mathrm{mg} / \mathrm{dL}, \mathrm{N}(\%)$ & $29(53.7)$ & $28(20.6)$ & $<0.0001$ & 4.474 & $2.273-8.809$ \\
\hline \multicolumn{6}{|c|}{$\begin{array}{l}\text { SAH: systemic arterial hypertension; CAD: coronary artery disease; COPD: chronic obstructive pulmonary disease; CHF: congestive heart failure; } \\
\text { CRF: chronic renal failure; CT pulmonary: pulmonary parenchymal alterations in chest computed tomography; hsCRP: high-sensitive C-reactive } \\
\text { protein; hsTnI: high-sensitivity troponin I; INR: international normalized ratio; Leukocytosis means white blood cell count } \geq 10500 / L ; \text { Lymphocytopenia, } \\
\text { lymphocyte count }<900 / L ; \text { thrombocytopenia, platelet count }<150000 / L \text {. }\end{array}$} \\
\hline
\end{tabular}


coronary artery disease (CAD), stroke, congestive heart failure (CHF), and systemic arterial hypertension (SAH), as well as lymphocytopenia, higher levels of creatinine, D-dimer, INR, and hTnI upon hospital admission than those who survived and were discharged.

The average length of stay was not different between those who died and those who were discharged (14 \pm 13 days vs $14 \pm 11$ days; $P=0.174$ ).

No difference in mortality was found between men and women ( $27.7 \%$ vs $28.7 \%$, respectively; $\mathrm{P}=0.872$ ).

Table 2 shows that during hospitalization, more patients who died received invasive $M V$, RRT, vasoactive amine, and transfer to the ICU compared to those who were discharged.

There was no difference between those who died and those who were discharged with regard to the development of acute LVSD during hospitalization (10.6\% vs $2.7 \%$; $\mathrm{P}=0.109$ ).

Table 3 shows that, in the binary logistic regression analysis, it was found that elevated hTnI levels upon hospital admission, and the need for invasive MV during hospitalization, increased the chance of hospital mortality.

Patients with hTnI elevation upon hospital admission are more likely to have previous CHF $(17.2 \%$ vs $2.7 \%$; $\mathrm{P}=0.010 ; \mathrm{OR}=7.431 ; 95 \% \mathrm{CI}=1.661-33.243), \mathrm{COPD}(20.7 \%$ vs $6.4 \% ; \mathrm{P}=0.029 ; 95 \% \mathrm{CI}=1.179-12.497$ ), and the need for invasive MV during hospitalization ( $55.2 \%$ vs $21.8 \%$; $\mathrm{P}=0.001 ; \mathrm{OR}=4.410 ; 95 \% \mathrm{CI}=1.865-10.426$ ) as compared to patients who have normal hTnI levels.

No difference was found in relation to previous history of stroke ( $13.3 \%$ vs $9.1 \%$; $\mathrm{P}=0.49)$, CAD (27.6\% vs $23.6 \%$;
$\mathrm{P}=0.63)$, $\mathrm{SAH}$ (72.4\% vs $60,0 \%$; $\mathrm{P}=0.28)$, diabetes $(44.8 \%$ vs $33.6 \% ; \mathrm{P}=0.28$ ), and the development of acute LVSD ( $7.7 \%$ vs $6.3 \% ; \mathrm{P}=1.0$ ) during hospitalization between patients with high and normal TnI levels, respectively, upon hospital admission.

\section{Discussion}

According to the findings of this study, it is suggested that, in a selected population of symptomatic hospitalized adult patients with a confirmed diagnosis of COVID-19, the presence of myocardial injury, characterized by high levels of hTnI upon hospital admission, is associated with an increased risk of death.

There is growing evidence of an association between myocardial injury and an increased risk of death in patients with COVID-19. Several studies involving patients with confirmed COVID-19 have implied the elevation of serum hTnI not only with mortality, ${ }^{4,12-19}$ but also criticality. $8,14,16$

Although some investigators ${ }^{4,14,16,18}$ have found an association between inflammatory biomarkers with poor prognosis, in the present study, only higher levels of hTnI were independently associated with mortality (table 3).

Another finding of this study, unlike others, ${ }^{6,12,13,18}$ is that the presence of cardiovascular comorbidities was not independently associated with higher mortality (Table 3). In addition, it was demonstrated (table 3 ) that there was no independent association between older age ( $>60$ yearold) and mortality as has been demonstrated in other studies. ${ }^{12,14,16}$ In the present study, the mortality of patients aged $\geq 60$ years of age was $37.8 \%$, while the mortality of

Table 2 - Factors involved in mortality during hospitalization in univariate analysis

\begin{tabular}{|c|c|c|c|c|c|}
\hline Characteristics & $\begin{array}{c}\text { Discharged } \\
(\mathrm{N}=138)\end{array}$ & Died $(N=54)$ & p-value & OR & $95 \% \mathrm{CI}$ \\
\hline LVSD, N (\%) & $2(2.7)$ & $5(10.6)$ & 0.109 & 4.226 & $0.785-22.758$ \\
\hline RRT, N (\%) & $2(1.4)$ & $11(20.4)$ & $<0.0001$ & 17.395 & $3.710-81.562$ \\
\hline Transfer to ICU, N (\%) & $17(12.3)$ & $19(35.2)$ & 0.001 & 3.864 & $1.817-8.219$ \\
\hline Vasoactive amine, $\mathrm{N}(\%)$ & $20(14.5)$ & $30(55.6)$ & $<0.0001$ & 7.375 & $3.604-15.092$ \\
\hline MV, N (\%) & 16 (11.6) & 41 (75.9) & $<0.0001$ & 24.048 & $10.667-54.214$ \\
\hline
\end{tabular}


Table 3 - Results of binary regression analysis

\begin{tabular}{lccc}
\hline Characteristics & OR & 95\% CI & p-value \\
\hline Age $\geq 60$ years & 6.305 & $0.501-79.403$ & 0.154 \\
CAD & 8.093 & $0.944-69.376$ & 0.056 \\
Stroke & 13.980 & $0.327-597.291$ & 0.169 \\
CHF & 8.567 & $0.426-172.445$ & 0.161 \\
INR $>1.3$ & 6.221 & $0.765-50.613$ & 0.087 \\
D-dimer $>500 \mathrm{ng} / \mathrm{mL}$ & 1.507 & $0.034-66.740$ & 0.832 \\
Creatinine $>1.3 \mathrm{mg} / \mathrm{dL}$ & 1.206 & $0.196-7.404$ & 0.840 \\
hTnI $>34 \mathrm{pg} / \mathrm{mL}$ & 9.504 & $1.281-70.528$ & 0.028 \\
CT pulmonary alterations $>50 \%$ & 3.878 & $0.502-29.943$ & 0.194 \\
MV & 46.691 & $2.360-923.706$ & 0.012 \\
Vasoactive amine & 0.843 & $0.113-6.262$ & 0.867 \\
Transfer to ICU & 3.231 & $0.153-68.109$ & 0.451 \\
RRT & 10.175 & $0.800-129.444$ & 0.074 \\
\hline
\end{tabular}

CAD: coronary artery disease; CHF: congestive heart failure; CT pulmonary: pulmonary parenchymal alterations in chest computed tomography; hTnI: high-sensitivity troponin I; MV: invasive mechanical ventilation; ICU: intensive care unit; RRT: renal replacement therapy.

patients with higher hTnI was $69.4 \%$ (data not shown). In other words, it is suggested that myocardial injury upon hospital admission is much more dangerous than older age or cardiovascular comorbidities in the population with COVID-19 reported in this study.

Therefore, according to the findings of the present study and data collected from recent literature, it seems clear that the presence of myocardial injury in hospitalized adult patients with COVID-19 was associated with an increased risk of death. The pathophysiological substrate of myocardial injury produced by SARS-CoV-2 is not yet well defined, but it is speculated that an imbalance between myocardial demand and supply due to tachycardia, hypotension, and hypoxemia resulting in type 2 myocardial infarction, acute coronary syndrome due to a virally induced thrombotic and inflammatory state, microvascular dysfunction due to diffuse microthrombi or vascular injury, stress-related cardiomyopathy (Takotsubo syndrome), nonischemic myocardial injury due to hyperinflammatory cytokine storm, or a direct viral cardiomyocyte toxicity and myocarditis may be involved. 5,20
Although no association was found between a rise in troponin upon hospital admission and the development of acute LVSD, it must be taken into account that there was no analysis of the evolution of hTnI levels during hospitalization.

Another finding of the present study is the independent association of the need for invasive MV during hospitalization, and an increased chance of death. Patients who required MV had a much higher chance of death when compared to those who did not require MV (Table 2 and Table 3). Of 57 patients that needed MV, 41 died (71.9\%). This association has been reported by other studies, which have demonstrated that the mortality involved in the need for invasive MV varied from $33.9 \%$ to $90 \%$. ${ }^{16,21-24}$

The finding of an association between the need for MV and increased mortality seems obvious, as it implies greater severity of the disease, especially acute respiratory distress syndrome. In the studied population, patients who required MV present greater lung injury (40.4\% vs $21.8 \%$; $\mathrm{P}=0.013$ ), higher hTnI (40\% vs $13.1 \% ; \mathrm{P}=0.001)$, a greater need for ECMO (5.3\% vs 0\%; P = 0.025), and RRT $(21.1 \%$ vs $0.7 \%$; $\mathrm{P}<0.0001$ ) than those that did not require MV. 


\section{Limitations}

Like any retrospective study, this study suffers from the lack of systematization in the registration of clinical and laboratory characteristics of patients, implying that the associations between these data and mortality are suggestive and require external validation, involving a larger number of patients with similar clinical and laboratory characteristics.

Laboratory changes that occurred during the course of hospitalization were also not routinely analyzed, which could somehow modify the results of the study.

This is a study of a quaternary heart center, which increases the chance of involving more patients with heart diseases, although no difference was found in the presence of previous $\mathrm{CAD}$, stroke, chronic kidney dysfunction, SAH, diabetes or the development of acute LVSD among those with and without myocardial injury.

\section{Conclusions}

In a selected population of symptomatic hospitalized adult patients with COVID-19, the presence of myocardial injury upon hospital admission was independently associated with increased mortality. Therefore, it was suggested that hospitalized COVID-19 patients should have troponin levels measured routinely upon hospital admission in order to stratify the risk of death.

\section{References}

1. World Health Organization. WHO Coronavirus Disease (COVID-19) Dashboard [Internet]. Geneva: World Health Organization; 2021 [cited 2021 Jun 18]. Available from: https://covid19.who.int/

2. Machhi J, Herskovitz J, Senan AM, Dutta D, Nath B, Oleynikov MD, et al. The Natural History, Pathobiology, and Clinical Manifestations of SARS-CoV-2 Infections. J Neuroimmune Pharmacol. 2020;15(3):359-86. doi: 10.1007/s11481-020-09944-5.

3. Nishiga M, Wang DW, Han Y, Lewis DB, Wu JC. COVID-19 and Cardiovascular Disease: From Basic Mechanisms to Clinical Perspectives. Nat Rev Cardiol. 2020;17(9):543-58. doi: 10.1038/s41569-020-0413-9.

4. Zhou F, Yu T, Du R, Fan G, Liu Y, Liu Z, et al. Clinical Course and Risk Factors for Mortality of Adult Inpatients With COVID-19 in Wuhan, China: A Retrospective Cohort Study. Lancet. 2020;395(10229):1054-62. doi: 10.1016/S0140-6736(20)30566-3.

5. Figueiredo Neto JA, Marcondes-Braga FG, Moura LZ, Figueiredo AMES, Figueiredo VMES, Mourilhe-Rocha R, et al. Coronavirus Disease 2019 and the Myocardium. Arq Bras Cardiol. 2020;114(6):1051-57. doi: 10.36660/ abc. 20200373.

\section{Author contributions}

Conception and design of the research: Rocha ASC, Volschan A, Coelho RPS, Thielmann DCA. Acquisition of data: Rocha ASC, Volschan A. Analysis and interpretation of the data: Rocha ASC, Volschan A, Campos LAA, Thielmann DCA, Ferreira CALC, Colafranceschi AS. Statistical analysis: Rocha ASC, Volschan A. Writing of the manuscript: Rocha ASC, Volschan A. Critical revision of the manuscript for intellectual content: Rocha ASC, Volschan A, Campos LAA, Thielmann DCA, Ferreira CALC, Colafranceschi AS.

\section{Potential Conflict of Interest}

No potential conflict of interest relevant to this article was reported.

\section{Sources of Funding}

There were no external funding sources for this study.

\section{Study Association}

This study is not associated with any thesis or dissertation work.

\section{Ethics approval and consent to participate}

This study was approved by the Ethics Committee of the Hospital Pró-Cardíaco under the protocol number 31543220.1.0000.5533. All the procedures in this study were in accordance with the 1975 Helsinki Declaration, updated in 2013. Informed consent was obtained from all participants included in the study.

6. Souza CDF, Leal TC, Santos LG. Does Existence of Prior Circulatory System Diseases Accelerate Mortality Due to COVID-19? Arq Bras Cardiol. 2020;115(1):146-47. doi: 10.36660/abc.20200486

7. Bonow RO, Fonarow GC, O'Gara PT, Yancy CW. Association of Coronavirus Disease 2019 (COVID-19) With Myocardial Injury and Mortality. JAMA Cardiol. 2020;5(7):751-753. doi: 10.1001/ jamacardio.2020.1105

8. Almeida GLG Jr, Braga F, Jorge JK, Nobre GF, Kalichsztein M, Faria PMP, et al. Prognostic Value of Troponin-T and B-Type Natriuretic Peptide in Patients Hospitalized for COVID-19. Arq Bras Cardiol. 2020 Oct;115(4):660-66. doi: 10.36660/abc.20200385.

9. Farias LPG, Fonseca EKUN, Strabelli DG, Loureiro BMC, Neves YCS, Rodrigues TP, et al. Imaging findings in COVID-19 pneumonia. Clinics. 2020;75:e2027. doi: 10.6061/clinics/2020/e2027.

10. Costa IBSDS, Rochitte CE, Campos CM, Barberato SH, Oliveira GMM, Lopes MACQ, et al. Cardiovascular Imaging and Interventional Procedures in Patients with Novel Coronavirus Infection. Arq Bras Cardiol. 2020;115(1):111-26. doi: 10.36660/abc.20200370. 
11. Charlson ME, Pompei P, Ales KL, MacKenzie CR. A New Method of classifying Prognostic Comorbidity in Longitudinal studies: Development and Validation. J Chronic Dis. 1987;40(5):373-83. doi: 10.1016/0021-9681(87)90171-8

12. Du RH, Liang LR, Yang CQ, Wang W, Cao TZ, Li M et al. Predictors of Mortality for Patients With COVID-19 Pneumonia Caused by SARSCoV-2: A Prospective Cohort Study. Eur Respir J. 2020;55(5):2000524. doi: 10.1183/13993003.00524-2020.

13. Guo T, Fan Y, Chen M, Wu X, Zhang L, He T, et al. Cardiovascular Implications of Fatal Outcomes of Patients With Coronavirus Disease 2019 (COVID-19). JAMA Cardiol. 2020;5(7):811-818. doi: 10.1001/ jamacardio.2020.1017.

14. Shi S, Qin M, Shen B, Cai Y, Liu T, Yang F, et al. Association of Cardiac Injury With Mortality in Hospitalized Patients With COVID-19 in Wuhan, China. JAMA Cardiol. 2020;5(7):802-10. doi: 10.1001/ jamacardio.2020.0950.

15. Qin JJ, Cheng X, Zhou F, Lei F, Akolkar G, Cai J, et al. Redefining Cardiac Biomarkers in Predicting Mortality of Inpatients With COVID-19. Hypertension. 2020;76(4):1104-12. doi: 10.1161/ HYPERTENSIONAHA.120.15528.

16. Petrilli CM, Jones SA, Yang J, Rajagopalan H, O'Donnell L, Chernyak Y, et al. Factors Associated With Hospital Admission and Critical Illness Among 5279 People With Coronavirus Disease 2019 in New York City: Prospective Cohort Study. BMJ. 2020;369:m1966. doi: 10.1136/bmj.m1966.

17. Bardají A, Carrasquer A, Sánchez-Giménez R, Lal-Trehan N, DelMoral-Ronda V, Peiró ÓM, et al. Prognostic Implications of Myocardial Injury in Patients With and Without COVID-19 Infection Treated in a University Hospital. Rev Esp Cardiol. 2021;74(1):24-32. doi: 10.1016/j recesp.2020.08.009
18. Shoar S, Hosseini F, Naderan M, Mehta JL. Meta-analysis of Cardiovascular Events and Related Biomarkers Comparing Survivors Versus Non-survivors in Patients With COVID-19. Am J Cardiol. 2020;135:50-61. doi: 10.1016/j.amjcard.2020.08.044.

19. Martins-Filho PR, Barreto-Filho JAS, Santos VS. Myocardial Injury Biomarkers and Cardiac Complications Associated with Mortality in Patients with COVID-19. Arq Bras Cardiol. 2020;115(2):273-77. doi: 10.36660/abc.20200372.

20. Madjid M, Safavi-Naeini P, Solomon SD, Vardeny O. Potential Effects of Coronaviruses on the Cardiovascular System: A Review. JAMA Cardiol. 2020;5(7):831-40. doi: 10.1001/jamacardio.2020.1286.

21. Docherty AB, Harrison EM, Green CA, Hardwick HE, Pius R, Norman L, et al. Features of 20133 UK Patients in Hospital With Covid-19 Using the ISARIC WHO Clinical Characterisation Protocol: Prospective Observational Cohort Study. BMJ. 2020;369:m1985. doi: 10.1136/bmj.m1985.

22. Richardson S, Hirsch JS, Narasimhan M, Crawford JM, McGinn T, Davidson KW, et al. Presenting Characteristics, Comorbidities, and Outcomes Among 5700 Patients Hospitalized With COVID-19 in the New York City Area. JAMA. 2020;323(20):2052-59. doi: 10.1001/jama.2020.6775.

23. Gavin W, Campbell E, Zaidi SA, Gavin N, Dbeibo L, Beeler C, et al. Clinical Characteristics, Outcomes and Prognosticators in Adult Patients Hospitalized With COVID-19. Am J Infect Control. 2021;49(2):158-65. doi: 10.1016/j.ajic.2020.07.005.

24. Wu C, Chen X, Cai Y, Xia J, Zhou X, Xu S, et al. Risk Factors Associated With Acute Respiratory Distress Syndrome and Death in Patients With Coronavirus Disease 2019 Pneumonia in Wuhan, China. JAMA Intern Med. 2020;180(7):934-43. doi: 10.1001/jamainternmed.2020.0994. 